\title{
Introduction to the special issue on monoamine oxidase $A$ and $B$ : eternally enigmatic isoenzymes
}

\author{
Jean Chen Shih ${ }^{1,2,3,4,5} \cdot$ Peter Riederer ${ }^{6,7} \cdot$ Wakako Maruyama $^{8} \cdot$ Makoto Naoi $^{8}$
}

Received: 13 August 2018 / Accepted: 22 August 2018 / Published online: 26 September 2018

(c) Springer-Verlag GmbH Austria, part of Springer Nature 2018

Mary L. C. Hare (later, Bernheim) first reported an enzyme catalyzing the oxidation of monoamines in 1928 (Hare, 1928), which was later named monoamine oxidase (MAO). Since then, the structure, function and regulation of expression have been intensively studied. MAO A and B showed substrate and inhibitor specificities. These two forms of MAO were clearly identified as two independent proteins encoded by different genes in 1988. These MAO genes exhibit identical intron and exon organization derived from duplication of a common ancestral gene millions of years ago, thus they are termed MAO A and B isoenzymes. MAO A and MAO B are localized in distinct cell types and tissues, with specific functions in the brain and peripheral tissues. MAO is associated with the regulation of monoamine neurotransmitters and is important for the development and maintenance of neuronal architecture and circuits. Further, MAO regulates motor coordination, cognition, multiple behaviors, mood and emotion, and recently has been implicated in the diagnosis and therapy of cancers. Studies with MAO A and B knockout mice demonstrate that MAO A gene and environmental interaction are involved in emotional behaviors such as aggression, anxiety,

Jean Chen Shih

jcshih@usc.edu

Peter Riederer

peter.riederer@uni-wuerzburg.de

Wakako Maruyama

maruyama@dpc.agu.ac.jp; mwakako@sc.starcat.ne.jp

Makoto Naoi

mnaoi@dpc.agu.ac.jp; mnaoi@quartz.ocn.ne.jp

1 Department of Pharmacology and Pharmaceutical Sciences, School of Pharmacy, University of Southern California, Los Angeles, CA, USA

2 Department of Cell and Neurobiology, Keck School of Medicine, University of Southern California, Los Angeles, CA, USA

3 Norris Comprehensive Cancer Center, Keck School of Medicine, University of Southern California, Los Angeles, CA, USA autism, impulsivity, and anti-social behaviors. MAO A and $\mathrm{B}$ inhibitors were developed as antidepressants and L-DOPA adjuvant. Mechanistic investigations have yielded novel therapeutic strategies including neuroprotection in aging, neurodegenerative and mental disorders. MAO has been shown to play a decisive role in cellular death and survival by its involvement in cellular signaling pathways and the mitochondrial apoptosis cascade.

Significant progresses in MAO research have been made through continuous discussion and collaboration among scientists from different countries and research fields. The "Amine Oxidase Symposium" organized every 2 years worldwide has given us valuable opportunities to advance MAO research and build close lifelong friendships. As one generation of MAO researchers, we summarized our discoveries on MAO and pose challenging unanswered questions for the next generation to discover. Here, we have collectively merged historical discoveries with our latest findings on MAO. The present and future prospects of MAO inhibitors were reviewed. We hope that this special issue on

4 USC-Taiwan Center for Translational Research, University of Southern California, Los Angeles, CA, USA

5 College of Pharmacy, Taipei Medical University, Taipei, Taiwan

6 University Hospital Wuerzburg, Clinic and Policlinic for Psychiatry, Psychosomatics and Psychotherapy, Margarete Hoeppel-Platz 1, 97080 Wuerzburg, Germany

7 Department of Psychiatry, Research Unit for Psychiatry, University of South Denmark, JB Winslows Vej 18, 5000, Odense, Denmark

8 Department of Health and Nutrition, Faculty of Psychological and Physical Science, Aichi Gakuin University, 12 Araike, Iwasaki-cho, Nisshin, Aichi 320-0195, Japan 
MAO will be able to help the readers to find new concepts for future studies on MAO, based on what we have found.

Editors:

Jean Chen Shih, Peter Riederer, Wakako Maruyama and Makoto Naoi

\section{Reference}

Hare MLC (1928) Tyramine oxidase I. A new enzyme system in liver. Biochem J 22:968-979 